\title{
ON THE NATURE OF THE SUBSTANCE(S) PRODUCING PAIN IN \\ CONTRACTING SKELETAL MUSCLE: ITS BEARING ON THE PROBLEMS OF ANGINA PECTORIS AND INTERMITTENT CLAUDICATION ${ }^{1}$
}

\author{
By L. N. KATZ, E. LINDNER, AND H. LANDT \\ (From the Heart Station and the Cardiovascular Laboratory, Department of Physiology, \\ Michael Reese Hospital, Chicago)
}

(Received for publication March 30, 1935)

The mechanism of the production of pain in angina pectoris and intermittent claudication has been the subject of many studies during the last century and a half. Interest in this has recently been reawakened by the investigation of Lewis and his collaborators $(1,2)$. They showed that the continuous pain from contracting ischemic skeletal muscle of normal subjects is due not to vascular spasm but to the development by the contracting skeletal muscle of a factor producing pain, confirming a viewpoint proposed by the early workers in this field during the 18 th century. In this laboratory we have recently been concerned with the nature of this factor producing pain. Our previous results $(3,4,5)$ may be summarized as follows:

1. The factor producing pain accumulates in resting ischemic muscle as well as in contracting ischemic muscle, but at a much slower rate than in contracting muscle. The ratio between the two rates of accumulation is roughly proportional to the relative rates of energy exchange as indicated by $\mathrm{O}_{2}$ consumption and heat production (cf. Hill (6)).

2. The accumulation of the factor producing pain in contracting skeletal muscle which is not ischemic is accelerated ( $a$ ) by stasis brought on by experimental venous engorgement, $(b)$ by generalized anoxemia without stasis or ischemia, and $(c)$ by increasing the rate at which the muscle is exercised.

3. After the exercise is stopped and the circulation to the muscle has been restored to normal, the factor producing pain takes a much longer time to disappear than does the pain. If the exercise is performed soon after a previous period of exercise or after a period of stasis or ischemia,

${ }^{1}$ Aided by the Frederick K. Babson Fund for the Study of Diseases of the Heart and Circulation. less exercise is required to cause pain in the subsequent test with the same muscle. This suggests a pre-pain state in which the amount of the factor producing pain while more than present normally is insufficient to stimulate the pain end organs.

These observations, when viewed in the light of the work of Lewis (2), MacWilliam and Webster (7), Reid (8) and others, suggested to us that the factor producing pain was a diffusible chemical product formed in the muscle during catabolism and disposed of in the presence of oxygen during the recovery of the muscle. The evidence for this contention was not conclusive, and the experiments in the present report were performed to test the hypothesis more completely. In addition, the experiments were planned to determine more precisely the nature of the factor producing pain.

\section{The effect of varying the degree of circulatory} stasis on the amount of exercise required to cause pain

We have shown previously that circulatory stasis modifies the amount of exercise required to cause pain (4). In this study we attempted to analyze more quantitatively than hitherto the influence of the degree of circulatory stasis on the amount of exercise required to cause pain. This was necessary before we could investigate other phases of the problem.

Method. Four normal subjects were used in these experiments. All tests were carried out on the right arm with the subject in the prone position. The arm was supported at a right angle to the body, and the exercise consisted of clenching the hand around a special ergograph (c.f. Kissin (3)) 60 times per minute in time with a metronome. The cuff, which was placed around the upper arm, was suddenly inflated to the de- 
sired degree by connecting it to an air reservoir, from which the appropriate pressure was obtained. The pressures used in the cuff in each set of exercises were 40,80 and $120 \mathrm{~mm}$. $\mathrm{Hg}$; the latter, by actual observation, was higher than the systolic arterial pressure of these subjects. The subject was commanded to start his exercise immediately afterward and to inform the observer when pain began and when it became almost unbearable. These moments were timed by the observer with a stopwatch. Each set of exercises was run on the same day, allowing 30 to 45 minutes to elapse between tests, an interval which Lewis et al. (1) and we (Perlow, Markle and Katz (4)) have shown is adequate to restore conditions to normal. A control exercise with no compression was run before and after each group. The order of the exercises was varied in the different sets of tests, and no exercise was run for more than 10 minutes.

Discussion of results. It will be seen from Figure 1 that in only one subject (E. L.) did pain

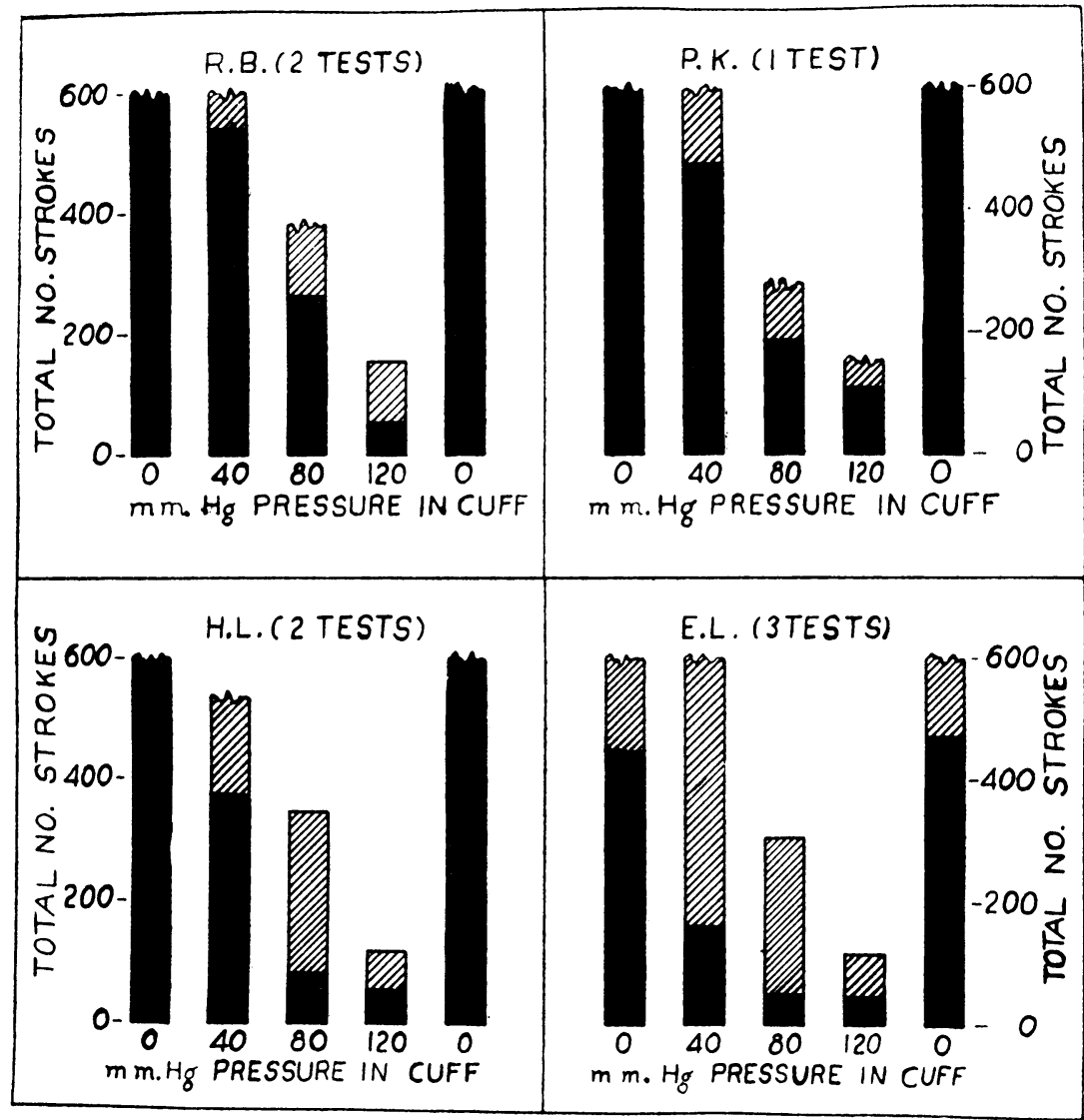

Fig. 1. Graphic Summary of Results with Varying Degrees of Circulatory Stasis.

Exercise at rate of 60 contractions per minute consisted of clenching the right hand around a special type of ergograph in time with a metronome. The ordinates give the total number of strokes, abscissae give the pressure in the cuff around the upper arm at the time of exercise. The height of each solid black block indicates the number of strokes required to cause the onset of mild pain, the total height (both solid and cross hatched) of each block indicates the number of strokes required to cause the onset of almost unbearable pain. A jagged top to the block at a level of 600 denotes that the pain indicated did not develop in the 10 minutes during which exercise was performed. A jagged top to the block below the level of 600 denotes that the pain indicated did not develop because the exercise had to be terminated because of neuromuscular fatigue. Discussion is in the text. 
appear in that 10 minute period when the circulation was free. Mild or almost unbearable pain whenever these could be evaluated, appeared sooner, as the degree of compression was increased. Raising the pressure the $40 \mathrm{~mm}$. $\mathrm{Hg}$ from 80 to 120 had less effect on the onset of mild pain than raising it the $40 \mathrm{~mm}$. $\mathrm{Hg}$ from 0 to 40 or from 40 to 80 . In E. L., raising the pressure the $40 \mathrm{~mm}$. $\mathrm{Hg}$ from 80 to 120 had less effect on the onset of almost unbearable pain than raising it the $40 \mathrm{~mm}$. $\mathrm{Hg}$ from 40 to 80 .

In several subjects exercise was stopped in less than 10 minutes because of neuromuscular fatigue (cf. Laplace and Crane (9)), viz. at $40 \mathrm{~mm}$. in P. K. and H. L. and at $80 \mathrm{~mm}$. in R. B. When this occurred, it was found that greater degrees of circulatory stasis caused an earlier appearance either of fatigue (P. K.), or of almost unbearable pain (H. L. and R. B.). In these instances, the higher degrees of circulatory stasis had more influence on the production of pain than on the production of neuromuscular fatigue. The process causing pain, which depends on stimulation of the end-organs of afferent nerve, is more sensitive to increased circulatory stasis than is neuromuscular fatigue, which in these experiments depends on modification of the myoneural junction and of the efferent nerve endings.

The compression produced in these experiments has two effects. In the first place it slows the circulation by impeding the flow of blood in the veins; in fact no flow can occur until the pressure in the veins exceeds that in the cuff. This results in an accumulation in the tissues of those waste products which normally diffuse into the blood stream or which require an adequate supply of oxygen for their destruction. In the second place, it causes stagnation in the veins and capillaries of a variable amount of blood which comes into diffusion equilibrium with the tissues. This stagnation is least when the arteries are occluded simultaneously with the veins, viz. at 120 $\mathrm{mm} . \mathrm{Hg}$. In fact, the content of the blood under these circumstances should be about that in the unoccluded arm. The stagnation would be greatest at $80 \mathrm{~mm} . \mathrm{Hg}$ and intermediate at 40 .

An examination of the experimental results shows that the amount of blood stagnant in the arm with occluded vessels has no demonstrable effect on the onset of pain or of neuromuscular fatigue; at least the effect, if it exists, is obscured by the dominant influence of the retardation of the circulation. The results show further that the effect of compression is not a linear function of the degree of compression, for the higher degrees of compression have a disproportionately greater effect than the lesser degrees. This may be because (1) greater degrees of compression cause a disproportionately greater amount of circulatory slowing; or because (2) the greater slowing of the circulation has a disproportionately greater effect, at least in depriving the tissues of oxygen. Such a disproportionate effect Kissin (3) has shown does occur in anoxemia.

It follows from these results that venous obstruction alone will have little influence on the onset of pain but when combined with arterial obstruction will greatly enhance the effects of the latter. This factor is evidently important in the development of intermittent claudication in the presence of varicosities and of angina pectoris either with elevated pressure in the right auricle or with thrombosis of the coronary veins.

\section{Effect of rate of exercise at various degrees of circulatory slowing on the amount of exercise required to cause pain}

That rate of exercise modifies the time of onset of pain we had noted previously, but until we recently computed the results in relation to the amount of exercise we had not appreciated that the earlier onset of pain with faster rates of exercise was, in fact, accompanied by a decrease in the amount of exercise required to cause pain. In the previous experiments the exercises were carried out without any anoxemia or stasis. The effect in them we interpret as due to the decrease in the time available for recovery at the faster rates of exercise. Hence we should expect that the amount of exercise required to cause pain would decrease less at faster rates of exercise when circulatory slowing is present and would be unaltered by rate of exercise when circulatory standstill is induced. This expectation was tested in the present study.

Method. The arm-exercise was carried out, in principle, in the same way as in the preceding experiments. The rates of exercise were 30,60 , 90 and 120 strokes per minute. These exercises 
were carried out with the cuff pressure at 0 (no stasis), at 40 , and at $80 \mathrm{~mm}$. $\mathrm{Hg}$ and, to insure complete ischemia, at $140 \mathrm{~mm}$. Hg. Measurements were made at a single cuff pressure but at different rates of exercise on each subject on a given day. This was repeated on different days at the other cuff pressures. Thirty to forty-five minutes of rest were allowed between exercises, and the order of the different rates of exercise was varied. Usually the effect of the exercises was studied at only one cuff pressure on any one day in a given subject.
At any given rate of exercise employed circulatory slowing produced an effect in the same direction as can be seen by comparing the vertical blocks at a given rate of exercise for different cuff pressures (Figures 2 and 3 ). The effect of circulatory slowing was less marked, however, at the faster rates than at the slower. This indicates that the circulatory slowing operates on the recovery phase of exercise, which is reduced in duration at the faster rates of exercise.

As anticipated, the effect of rate of exercise was less marked at the higher degrees of venous

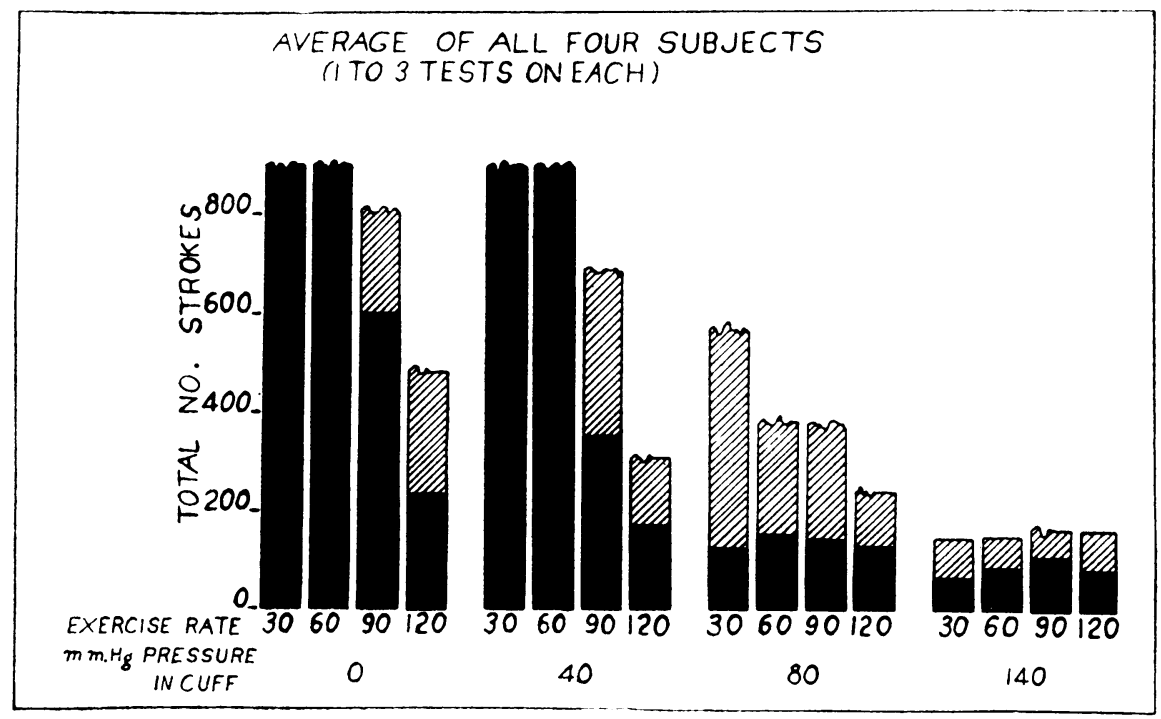

Fig. 2. Graphic Summary of Results on Four Subjects with Varying Rate of Exercise at Different Degrees of Circulatory Slowing.

The ordinates give the total number of strokes, the abscissae give the rate of exercise per minute (upper figures) and the pressure in the cuff around the upper arm at time of exercise (lower figures). Each group of four blocks is the average of all the tests carried out in all four subjects. Each of the four subjects was subjected to from 1 to 3 sets of tests, each set on a single day. The significance of the blocks is exemplified in Figure 1. A jagged line at the top of the vertical blocks at 900 denotes that the pain indicated did not develop by the time the exercise was stopped. A jagged line at the top of the vertical block below 900 denotes that the pain indicated did not develop because the exercise had to be stopped because of neuromuscular fatigue. A line at the top of the vertical block half jagged and half straight denotes that neuromuscular fatigue stopped about half of the tests and that pain of the type indicated stopped the other half. Discussed in text.

Discussion of results. Although the tests with different cuff pressures were carried out on different days instead of at one sitting, we confirmed the observation noted in the preceding section regarding the action of varying degrees of circulatory slowing on the onset of pain and added observations on the appearance of fatigue. occlusion than when the circulation was free, and the effect disappeared when circulatory standstill was produced. This is most clearly shown in the onset of mild pain, but the tendency was present also in the onset of almost unbearable pain. Assuming that the exercise per stroke was constant at different rates, these results indicate that the 
accumulated amount of the factor producing pain varies inversely as the time allowed for recovery. The time for recovery is less effective when the oxygen supply is limited, as in circulatory slowing, or when the oxygen supply is practically absent, as in circulatory standstill, than when the oxygen supply is adequate. Neuromuscular fatigue likewise appears after less exercise when the rate of exercise is faster, showing that it too depends on the time available for recovery from exercise. quired to cause pain there than previously. Preliminary experiments convinced us that exercising one group of muscles temporarily alters the amount of exercise required to cause pain in other groups of muscles, even when precautions are taken to prevent the transport of substances from the former to the latter. The experimental procedure had to be elaborated, therefore, in order to take account of this complicating factor.

Method. The usual arm-exercise with the er-

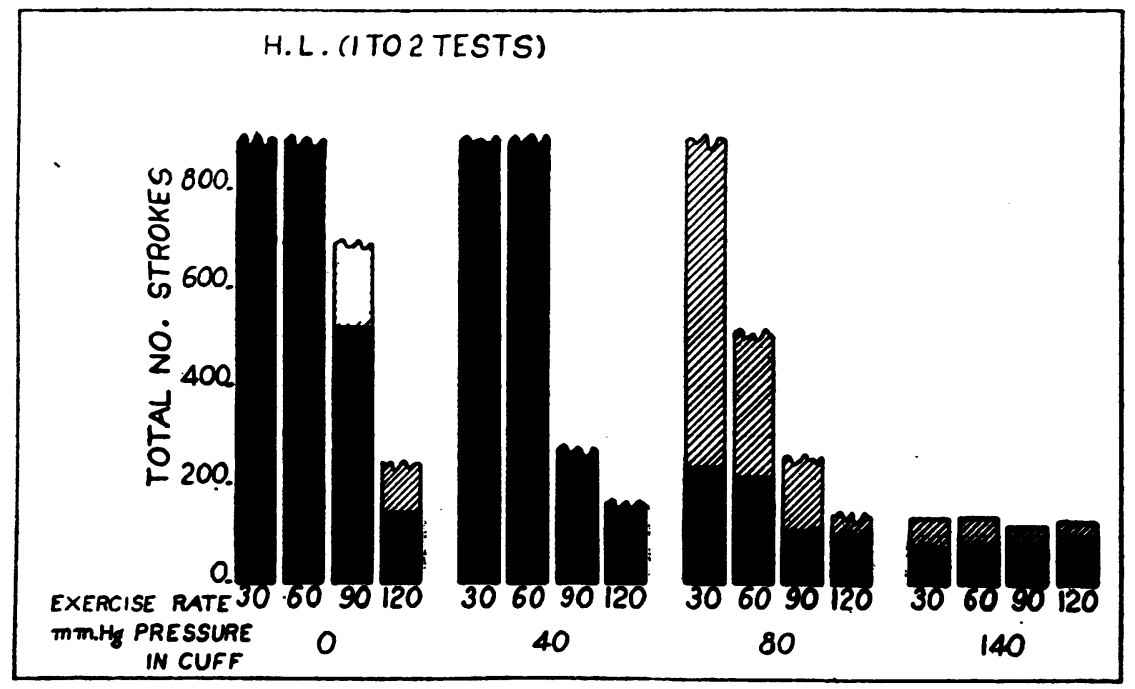

Fig. 3. Graphic Summary of Results in a Single Typical Subject with Varying Rate of Exercise at Different Degrees of Circulatory Stasis.

The significance of the blocks etc. is as in Figure 2. The results are the average of the tests carried out. Discussion is in the text.

The application of these results to the problem of pain in angina pectoris would suggest that the rate of the heart beat would be more important when coronary circulation insufficiency was advanced, and that pain would appear in such a case with less work by the heart.

\section{Evidence indicating that the factor producing pain is a substance which diffuses in and out of the blood stream}

No clear proof exists that the factor producing pain is a substance(s) which diffuses into and out of the blood stream. In principle, the proof of diffusion depends on finding that if one group of ischemic muscles undergoes exercise until pain appears, then if the blood from this limb has passed to another limb, less exercise will be re- gograph was carried out in 17 sets of 4 tests on normal subjects at 60 strokes per minute. Rest intervals ( 30 to 45 minutes) were allowed after each exercise, the sequence being varied in the different sets except that $(d)$ always followed (c) (vide infra). The total number of strokes necessary to bring on slight, moderate and almost unbearable pain was counted.

The four different conditions preceding the arm-exercise were as follows:

(a) Leg-exercise was provided on a frictionbrake bicycle ergometer at the rate of 60 strokes per minute. The legs were compressed at the upper thigh by raising the pressure in the cuff approximately to $220 \mathrm{~mm}$. Hg. Exercise was continued until moderately severe pain developed. The subject then walked to the bed preparatory to undertaking the arm-exercise. The pressure in 
the cuffs on the legs was then released. Twenty seconds later, when blood from the leg had arrived in the arm, the cuff on the arm was inflated $(160 \mathrm{~mm} . \mathrm{Hg}$ ) and the arm-exercise was begun. This we will refer to as exercise after trapping.

(b) For comparison, arm-exercise was carried out without previous leg-exercise, compression of the arm (160 mm. $\mathrm{Hg}$ ) being simultaneous with the onset of exercise. This we will refer to as the control for exercise after trapping.

(c) In order to prevent the action of the blood coming from the exercised legs from affecting the exercising arm, the arm was compressed (160 $\mathrm{mm} . \mathrm{Hg}$ ) before and during the leg-exercise and kept so until the end of the arm-exercise. A pressure of $160 \mathrm{~mm}$. $\mathrm{Hg}$ was found to be above the systolic pressure in the arm in all the subjects, even during the leg-exercise. This we will refer to as exercise without trapping. Continuous compression of the arm is required because blood cannot be shut off from all of the muscles of the leg and diffusible material could, therefore, be transported from the leg throughout the body.

(d) Since a preliminary period of ischemia lessens the amount of exercise required to cause pain (4) it was necessary to compare this effect with that in exercise without trapping. While resting in bed, the arm was kept compressed (160 $\mathrm{mm} . \mathrm{Hg}$ ) for 2 to 4 minutes, which was the time required for the onset of pain in the leg in the experiments on exercise without trapping and therefore the interval in these experiments during which the arm was compressed before starting arm-exercise. This we will refer to as the control for exercise without trapping. This exercise was carried out after the exercise without trapping.

Discussion of results. In order to ascertain the specific effect of blood from the exercised leg when trapped in the arm prior to exercise, free from other influences due to the leg-exercise, the following computations were made (cf. Table I).

Equation 1: (Onset of pain in control for exercise without trapping) minus (onset of pain in exercise without trapping) equals (onset of pain in arm due to factors following leg-exercise other than that mediated by the blood carried to the arm).

Equation 2: (Onset of pain in control for exercise after trapping) minus (onset of pain in exer-

\section{TABLE I}

Calculations used in a typical set of exercises, also shown graphically in Figure 5, to show how the effect of the diffusible pain-producing substance $(s)$ is determined.

Subject C. H.

\begin{tabular}{|c|c|c|c|}
\hline \multirow{2}{*}{ Type of exercise } & \multicolumn{3}{|c|}{$\begin{array}{c}\text { Onset of pain } \\
\text { (number of strokes) }\end{array}$} \\
\hline & Slight & Moderate & $\begin{array}{c}\text { Almost } \\
\text { unbear- } \\
\text { able }\end{array}$ \\
\hline 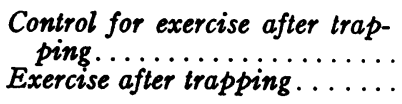 & $\begin{array}{l}92 \\
86\end{array}$ & $\begin{array}{l}118 \\
119\end{array}$ & $\begin{array}{l}147 \\
137\end{array}$ \\
\hline $\begin{array}{l}\text { A from Equation } 1 \ldots \ldots \\
\text { Control for exercise without } \\
\text { trapping } \\
\text { Exercise without trapping . . . . }\end{array}$ & $\begin{array}{r}-6 \\
90 \\
125\end{array}$ & $\begin{array}{l}+1 \\
109 \\
160\end{array}$ & $\begin{array}{r}-10 \\
130 \\
196\end{array}$ \\
\hline $\begin{array}{l}\text { B from Equation } 2 \ldots \ldots \\
A \text { minus } B \text { from Equa- } \\
\text { tion } 3 \ldots \ldots \ldots \ldots \ldots \ldots\end{array}$ & $\begin{array}{l}+35 \\
-41\end{array}$ & $\begin{array}{l}+51 \\
-50\end{array}$ & $\begin{array}{l}+66 \\
-76\end{array}$ \\
\hline
\end{tabular}

* Since leg-exercise was carried out for 2 minutes to produce pain in the legs in this test, the preliminary compression of the arm in this control was carried out for the same period before beginning the arm-exercise.

cise after trapping) equals (onset of pain in arm due to all factors following leg-exercise).

Equation 3: (Onset of pain in arm due to all factors following leg-exercise (from Equation 2)) minus (onset of pain in arm due to factors following leg-exercise other than that mediated by the blood carried to the arm (from Equation 1)) equals (onset of pain in arm due to factor following leg-exercise mediated by the blood carried to the arm).

Columns $I$ in Figure 4 show the scatter of results depicting the effect of factors following legexercise other than that mediated by the blood carried to the arm to be exercised, on the onset of slight, moderate and almost unbearable pain in the subsequent arm-exercise. Columns II of Figure 4 show the scatter of results depicting the combined effect of all factors following legexercise on the onset of the various degrees of pain in the subsequent arm-exercise. Columns III of Figure 4, derived in each set of exercises by subtracting algebraically the value in Column II from that in Column I, show the scatter of results depicting the effect of the factor following leg-exercise mediated by the blood carried from 


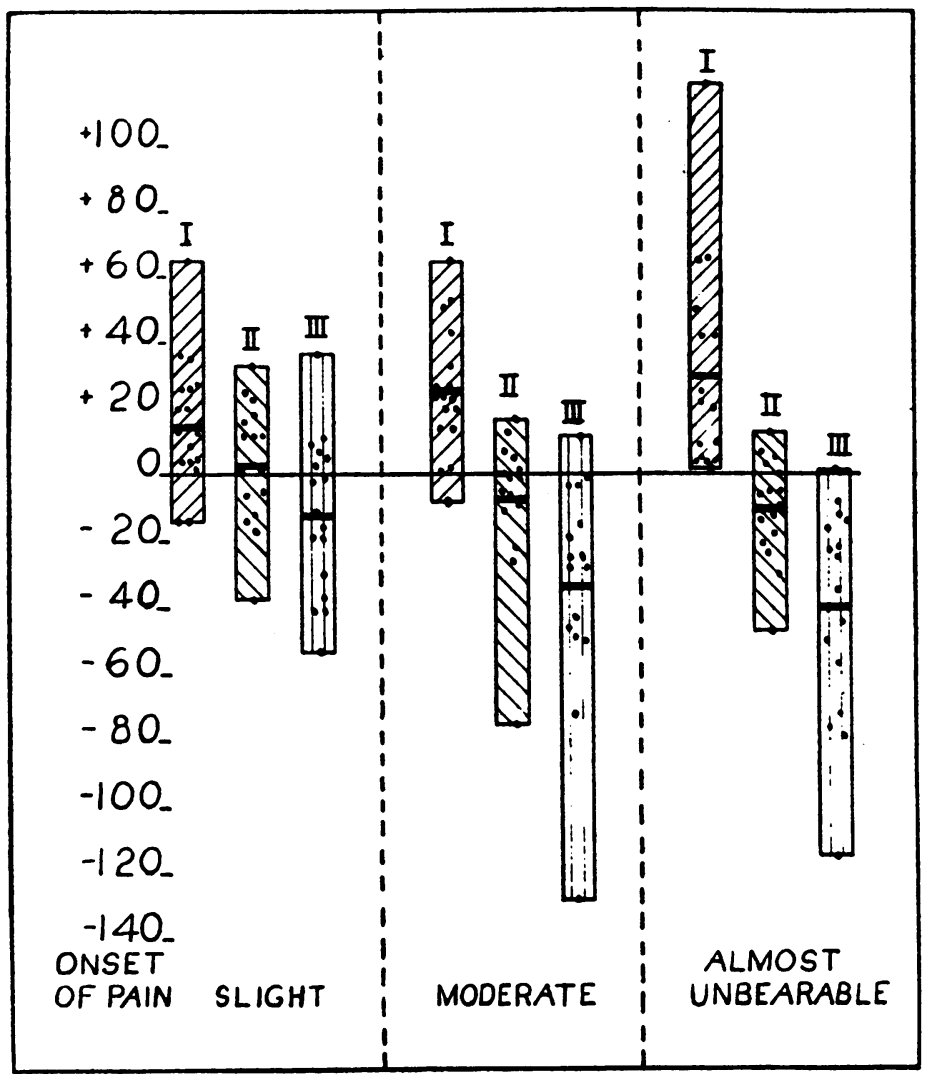

Fig. 4. Graphic Summary of Results in 17 Sets of Tests on 12 Normal Subjects, Showing the Effect on the Amount of Exercise ReQuired to Cause Pain in Exercise without Trapping (Colum I), In Exercise after Trapping (Colum II), AS Well as the Computed Effect of the Pain-Producing Substance(s) DifFusing into the Blood from the Exercising Legs and Diffusing out into the Muscles of the ARM When the Blood from the Legs is Trapped in the Arm to be Exercised (Colum III).

The columns are grouped for the onset of slight, moderate and almost unbearable pain. The ordinates show the effects in terms of total number of strokes; - means a decrease, and + , an increase in the number of strokes required to cause pain as compared with the controls for the subject. The manner of calculating each of these columns is given in the text: viz. Column I is determined by Equation 1: Column II by Equation 2; and Column III by Equation 3. Each column gives the range of scatter of the results in the 17 subjects. The results of the individual experiments are shown by the dots within the columns and the arithmetic average of the 17 tests in each column is shown by a horizontal line. Discussed in text.

the leg and trapped in the arm on the onset of various degrees of pain in the subsequent armexercise. The arithmetical average of each of these columns is shown by the horizontal heavy line within each column, and inspection will show that this is close to the median.
This figure, and also the summary of the four typical sets of exercise depicted in Figure 5, shows that exercising the legs to the point of moderately severe pain has a two-fold effect on the subsequent development of pain in the armexercise, most clearly seen with the severer de- 


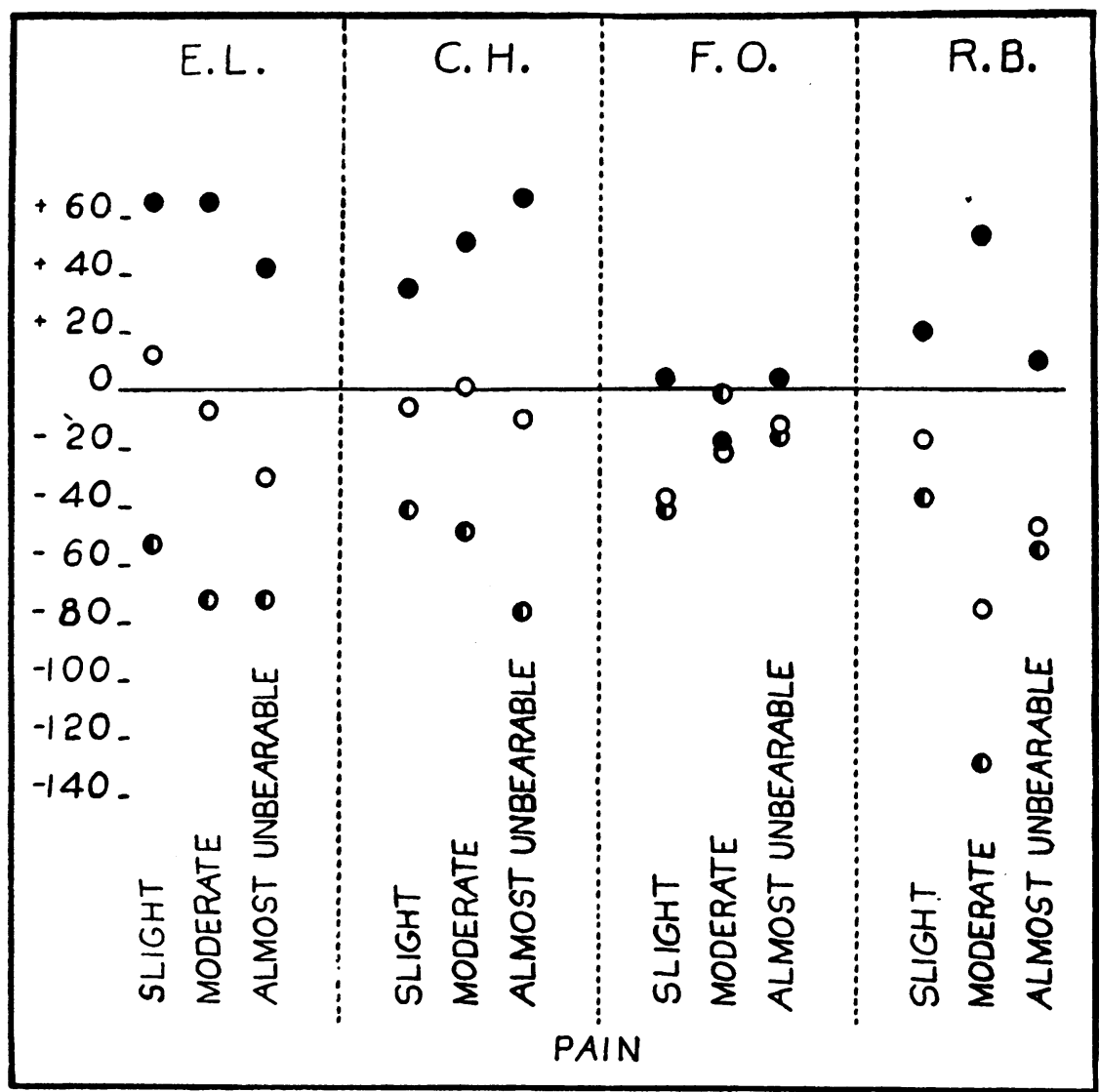

Fig. 5. Graphic Summary of Results in 4 Sets of Tests, Typical of All, Showing the Effect on the Amount of Exercise Required to Cause Pain in Exercise without Trapping (SOLID CIRCLES), IN Exercise after Trapping (OPEN Circles), as well as the Computed Effect of the Pain-Producing Substance(s) Diffusing into the Blood from the Exercising Legs and Diffusing out into the Muscle of the Arm When the Blood from the Legs was Trapped in the Arm to be Exercised (Half Solid, Half Open Circles).

The calculations are carried out in accordance with Equations 1,2 and 3 in the text. The ordinates show the effect in terms of total number of strokes; - means a decrease, and + , an increase in the number of strokes required to cause pain as compared with the control for the subject. Discussed in the text.

grees of pain in the arm. One action, the one not carried by way of the blood to the arm, tends to increase the amount of exercise required to cause pain, and the other action, that mediated by something transported by way of the blood to the arm, tends to decrease the amount of exercise required to cause pain. These actions do not appear clearly in every experiment but, considering the variables involved in such testing, these tendencies, it seems to us, are quite striking and surprisingly constant in the entire series. Considering the magnitude of the changes observed in most experiments, especially in the onset of the severer forms of pain, the facts as stated above seem to be beyond peradventure.

The manner in which the exercise without trapping augments the amount of exercise required to cause pain in the arm is not known. Several possibilities suggest themselves. It is possible that the effect is due to a change in the sensorium for perception of pain brought about by the mediation of the blood passing to the brain from the exercising 
legs. As mentioned above, it is impracticable to occlude the entire blood supply of the exercising legs. Hence diffusible materials from these parts, while kept out of the arm to be exercised, nevertheless can pass to other parts of the body, including the brain. The threshold of pain-perception may be raised in this way. A similarly bloodborne factor may affect the motor elements of the central nervous system so that the subsequent arm-exercise is carried out more efficiently as regards chemical change. Likewise an alteration may be produced by a reflex action either on the sensorium or motor elements of the central nervous system or on both. Still other possibilities exist which we are investigating. One thing, however, is certain, namely, that the augmentation of the amount of exercise required to cause pain in the exercise without trapping is not due to any blood-borne substance carried to the muscles to be exercised.

Making due allowance for this tendency to increase the amount of exercise required to cause pain, demonstrated in the exercise without trapping, in interpreting the results obtained in the exercise after trapping, brings out the action mediated by the blood on the muscles of the arm. This latter action is to decrease the amount of exercise required to cause pain. In other words, these results indicate that a substance(s) diffuses out of the exercising muscles of the leg into the blood stream, is carried by the blood stream through the lungs and to the muscles of the arm where it diffuses out and adds its effects to those of the substance(s) formed in the exercising arm. The net result is that less pain-producing substance(s) needs to be liberated by the exercising muscles of the arm to cause pain.

The fact that the blood exhibiting this additive effect is being carried to the arm past the lungs, would rule out the possibility that the pain-producing substance(s) was volatile, viz. $\mathrm{CO}_{2}$ or lack of $\mathrm{O}_{2}$, since the former is eliminated from the blood and the latter is supplied to it before the blood reaches the arm. It must, therefore, be some nonvolatile substance $(s)$ liberated during muscular activity. ${ }^{2}$

\footnotetext{
2 These results are consistent with the possibility that the action is not due to substances liberated by exercising muscle but to something removed from the blood during
}

If, as our results indicate, the pain-producing substance(s) is diffusible in and out of the blood stream, then it is probable that an attack of angina pectoris can be precipitated not only by the accumulation of pain-producing substance(s) liberated by the heart during its contraction but also in part by the transport to the heart of such pain producing substance(s) from other parts of the body. Thus, during exercise the pain producing substance(s) liberated from the skeletal muscles, and in states of relative anoxemia from all parts of the body, pours into the blood stream and diffuses into the heart where it is added to that liberated by the heart. Since it is well known that the chemical exchange during exercise, and to a lesser extent during anoxemia, is much more intense in the skeletal muscles than in the heart, this relationship is of practical importance. Thus, in angina of effort the attack may be due not only to the accumulation of pain-producing substance(s) liberated by the heart itself but to transport of this substance(s) to the heart from the exercising skeletal muscles.

\section{Is the pain-producing substance(s) acid in character?}

The demonstration in the foregoing experiments that the pain-producing substance(s) is diffusible and that it is not $\mathrm{CO}_{2}$ or lack of $\mathrm{O}_{2}$ limits the possibilities to two groups of substances formed during exercise, viz. (1) acid-metabolites such as lactic acid and some of the phosphoric acids and (2) non-acid metabolites such as creatinine. It is not practicable to test out the effects of these substances in man, and it is not easy to judge the reaction to pain in animals following injections. However, we felt that we could approach the problem by studying the effect which

exercise. This possibility is extremely unlikely, however, in view of facts previously established by us and by others in connection with the factor producing pain and in view of the well known fact that the alterations in exercise are due to liberated substances and not to deficiencies of substances, unless one is inclined to press the point that the appearance of one substance is accompanied by the disappearance of another. The only substance known to be removed from the blood in appreciable quantities is $\mathrm{O}_{2}$. This has been restored to the blood in our experiments during passage of the blood through the lungs. 
changing the acid-base balance of the blood and tissues has on the amount of exercise required to cause pain. After several preliminary tests with overventilation, ingestion of ammonium chloride, ingestion of sodium bicarbonate and hypercapnia, it was decided that the former two were unreliable, and the final experiments were confined to the latter two.

Methods. In the experiments with hypercapnia four normal subjects were tested, two tests being run on each. The arm-exercise was performed as described in the first section of this report. Before carrying out the exercise, the subjects inspired for as long as possible a prepared mixture of 10 to 11 per cent $\mathrm{CO}_{2}$ and 89 to 90 per cent $\mathrm{O}_{2}$ from a large Tissot spirometer. The blood was in this way kept saturated with oxygen and its $\mathrm{CO}_{2}$ content was definitely elevated. This hypercapnic blood was then trapped in the arm to be exercised by raising the pressure in the cuff on the upper arm to $160 \mathrm{~mm}$. Hg. Exercise was begun as soon as the cuff was inflated. Control exercise was run either before or after each test, a suitable rest period of 30 to 45 minutes being allowed between the two.

In the experiments with ingestion of sodium bicarbonate seven subjects were tested with the standard arm-exercise, rate 60 per minute and cuff pressure $160 \mathrm{~mm}$. $\mathrm{Hg}$. The control exercise in each subject was run during the morning of the first day. The exercise was repeated during the morning of the next day after the subject had ingested a total of 80 grains of $\mathrm{NaHCO}_{3}$, taken in 10 grain tablets every 2 hours during the waking period. The exercise was repeated on the following two mornings after the subject had taken a total of 240 and 360 grains of $\mathrm{NaHCO}_{3}$ respectively. In some subjects a control exercise was run in the morning 3 to 5 days after stopping the ingestion of $\mathrm{NaHCO}_{3}$. In W. R. a second test was run as follows: After a control exercise the subject ingested a total of 720 grains of $\mathrm{NaHCO}_{3}$ in 24 hours. A few hours after taking the last 220 grains a second exercise test was run, and a second control exercise was run four days later. The subjects tried to keep up their usual round of activities during the test period, aside from such variations as resulted from the reaction to the sodium bicarbonate.

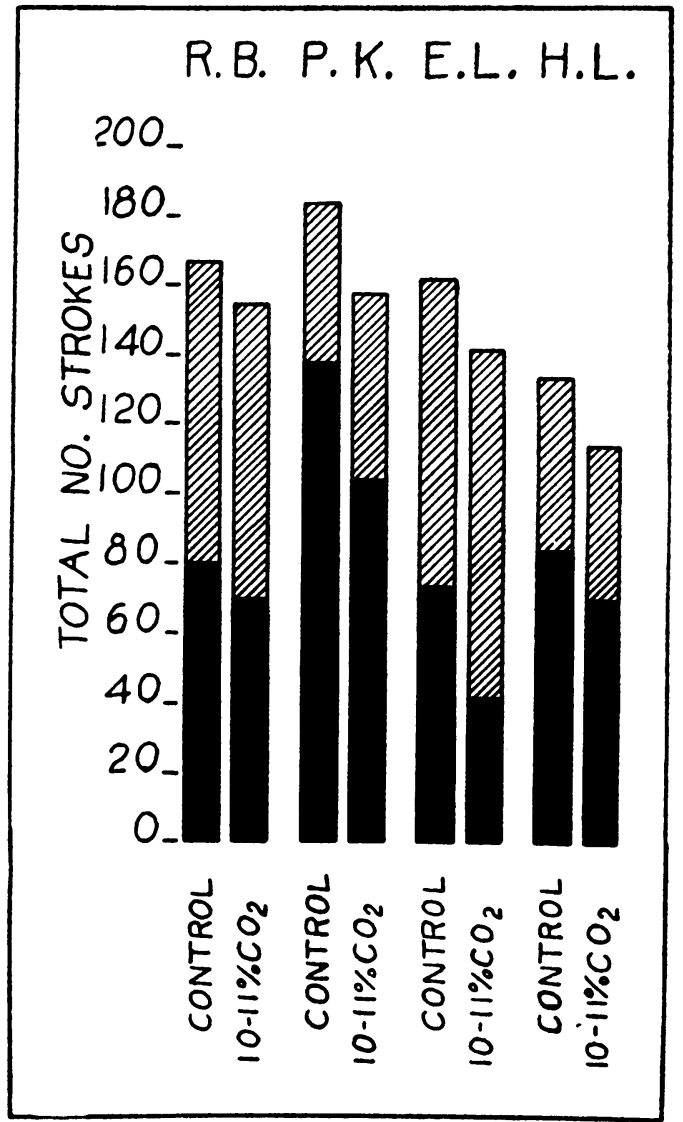

Fig. 6. Graphic Summary of Results in 4 Normal Individuals, Showing the EfFEct of Hypercapnia on the Amount of Exercise Required to Cause Pain.

Exercise at a rate of 60 contractions per minute was carried out as in the experiments shown in Figure 1, except that the cuff pressure was raised to $160 \mathrm{~mm}$. and the blood trapped in the arm contained an elevated $\mathrm{CO}_{2}$ content. The manner in which the blood $\mathrm{CO}_{2}$ content was elevated is described in the text. The columns show the average of two tests on each subject. The significance of the blocks etc. is as in Figure 1. The control exercises were run the same day either 30 to 45 minutes before or after the exercise with hypercapnic blood. Discussed in text.

Discussion of results. In Figure 6 is shown the effect of hypercapnia, and in Figure 7 the effect of ingestion of sodium bicarbonate. It will be seen that the presence of hypercapnic instead of normal blood in the arm to be exercised produced a definite and consistent decrease in the amount of exercise required to cause either slight or almost unbearable pain. No exception was found in the 8 experiments. Similarly, definite results in the opposite direction were obtained in the seven sub- 


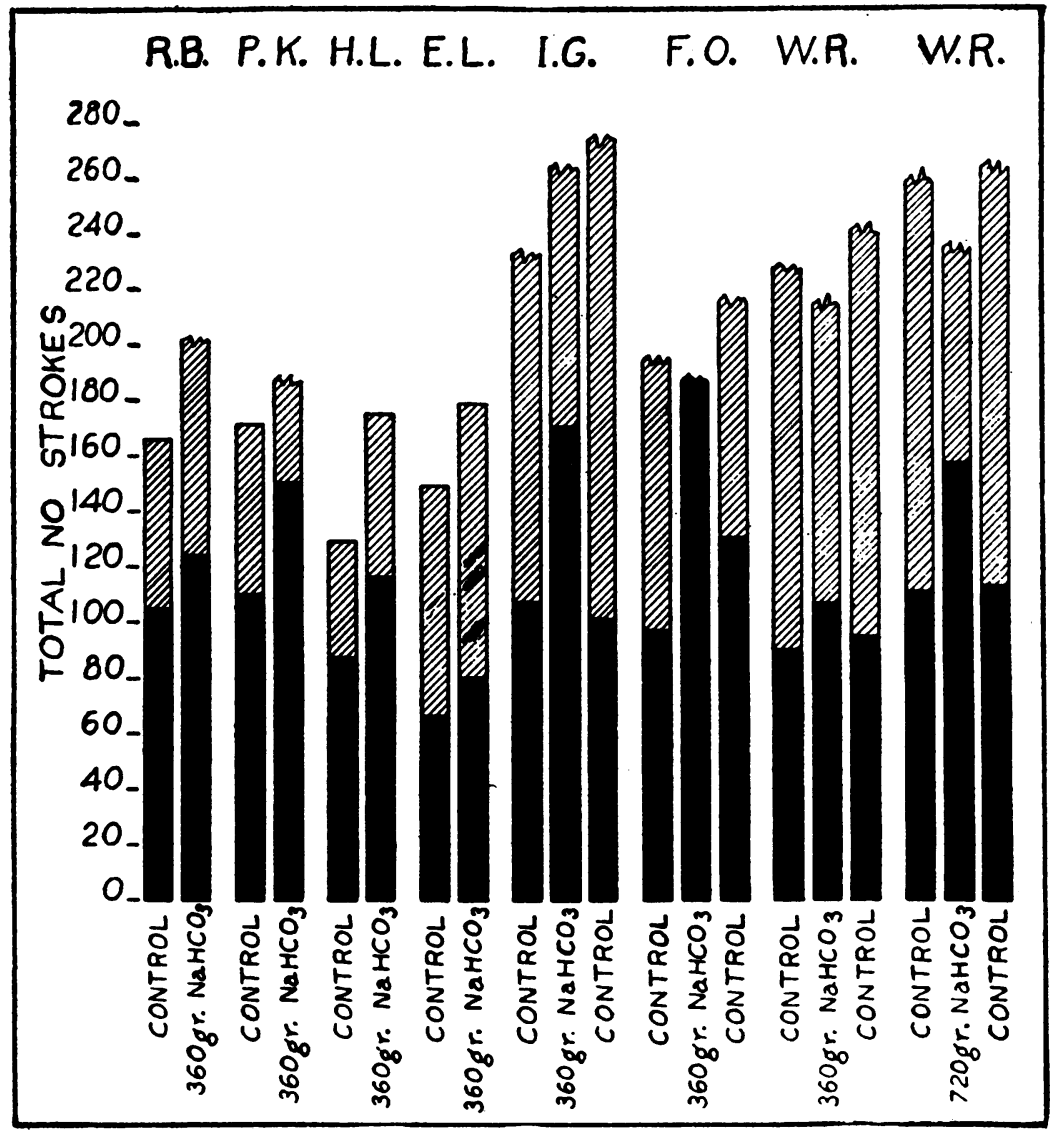

Fig. 7. Graphic Summary of Results in Seven Normal Subjects, Showing the EfFect of $\mathrm{NaHCO}_{3}$ Ingestion on the Amount of Exercise Required to Cause Pain.

The exercise was carried out in the same manner as in the experiments shown in Figure 6. The figure depicts the results at the time of maximum ingestion of $\mathrm{NaHCO}_{3}$ as compared with the controls taken on the day before, and 3 to 4 days after stopping ingestion of $\mathrm{NaHCO}_{3}$. The exact procedure is given in the text. The significance of the blocks etc. is as in Figure 1. Discussed in text.

jects tested with sodium bicarbonate; in all instances the amount of exercise required to cause pain was consistently increased by the sodium bicarbonate. This was only a temporary effect, as shown by the tendency to return to normal a few days after stopping the medication. In $W$. R. the effect was magnified by increasing the dosage of the $\mathrm{NaHCO}_{3}$ (see Figure 7). It is interesting to note that in several instances giving $\mathrm{NaHCO}_{3}$ changed the cause of cessation of the exercise from almost unbearable pain to neuromuscular fatigue (cf. R. B. and P. K., Figure 7). This effect is due probably to an earlier onset of neuromuscular fatigue (cf. F. O. and W. R., Figure
7) as well as to the delayed onset of almost unbearable pain (cf. H. L. and E. L.). These experiments show that the changes in the onset of neuromuscular fatigue are not only unrelated quantitatively to the changes in onset of pain but may be in opposite directions, as after giving $\mathrm{NaHCO}_{3}$.

There can be no question, in view of these experiments, that substances which can alter the acid-base balance of blood and tissues produce consistent changes, in the sense that an acid substance decreases and an alkaline substance increases the amount of exercise required to cause pain. While these results strongly suggest that 
the pain-producing substance(s) may be an acid metabolite, or at least one that operates by altering the acid-base balance in and around the endorgans for pain, they should not be considered as in any sense final proof of this. Several other possibilities suggest themselves. The action of the sodium bicarbonate may be on the sensorium or on the motor elements of the central nervous system; in the former case, raising the threshold to pain perception, in the latter increasing the efficiency of the exercise in terms of amount of chemical change. It is unlikely that the reverse is true in the experiments with hypercapnia since the blood and tissues other than those of the exercising arm are probably restored to normal before pain appears, unless one assumes a long lag in the wearing off of the hypercapnic effects. A much more likely possibility is that if the painproducing substance(s) is not acid in character then the $\mathrm{CO}_{2}$ and $\mathrm{NaHCO}_{3}$ alter the threshold of the endings for pain in the exercising arm and produce their effect in this manner. While these possibilities and probably others exist, we are strongly inclined to the view that the pain-producing substance is acid in character and propose this as a working hypothesis until evidence is advanced to disprove it. In fact, we are tempted to speculate, without warrant, that the substance may be lactic acid, the non-volatile acid formed in greatest amounts during exercise. It is interesting to note that Moore et al. (10) have found that acid substances, including lactic acid, when injected into the vessels of the limbs of animals produce responses indicating pain more easily than do other substances.

There is, in any event, no doubt that $\mathrm{CO}_{2}$ and $\mathrm{NaHCO}_{3}$ have an additive action with the pain producing substance(s); the $\mathrm{CO}_{2}$ acting in a positive, and the $\mathrm{NaHCO}_{3}$ in a negative manner. This action appears to be on the acid-base balance, in that the effect may be produced by altering the buffering capacity of the tissues, by altering the $\mathrm{pH}$ of the tissues, or in both ways.

The possibility of negative summation of the $\mathrm{NaHCO}_{3}$ with the pain-producing substance was tested out on patients with intermittent claudication and with angina pectoris in cooperation with Drs. Bohning, Gutman and Perlow. This experiment was suggested to us by Dr. Emanuel Lib- man when discussing our results with him. The results will be published later. Suffice it to say that the ingestion of large quantities of $\mathrm{NaHCO}_{3}$ produced some relief of pain in patients with intermittent claudication and with angina pectoris; this relief was not obtained with placebos used for control. This inference was based both on an estimate of the patient's subjective reactions and on the effect of test exercises. The therapy was in no way a cure, but some relief seemed to be obtained in most of the subjects.

Our results with $\mathrm{NaHCO}_{3}$ in the exercise of the arm lends some support to the view expressed by Laplace and Crane (9) that in some instances the failure to develop anginal pain or pain in intermittent claudication may be due to the earlier onset of slight degrees of neuromuscular (and muscular) fatigue imposing a restriction on the work of the heart or muscles and so limiting the accumulation of the pain-producing substances $(\mathrm{s})$. This may be one mode of action of $\mathrm{NaHCO}_{3}$. For example, in F. O., Figure 7, the $\mathrm{NaHCO}_{3}$ caused so early an onset of neuromuscular fatigue in relation to the appearance of pain that the exercise was terminated by fatigue before any pain appeared.

\section{$V$. Does training modify the time of appearance of pain in exercising muscle?}

In the course of this study we had occasion to compare in the two arms the amount of exercise required to cause pain in seven normal subjects. All were right handed, except F. O. who was ambidextrous. The standard arm-exercise was carried out as in the previous tests at the rate of 60 per minute and with cuff pressure $160 \mathrm{~mm}$. $\mathrm{Hg}$. The exercise of the two arms was carried out within 30 to 45 minutes of each other.

The results are shown graphically in Figure 8 and are in accord with those reported by Laplace and Crane (9). The left arm in all instances required less exercise to cause pain and neuromuscular fatigue than did the right. The reason for reporting this observation here is that it may have an important bearing on intermittent claudication and angina pectoris. The difference in the two arms is undoubtedly associated with training, since clenching of the hand had been carried out much 


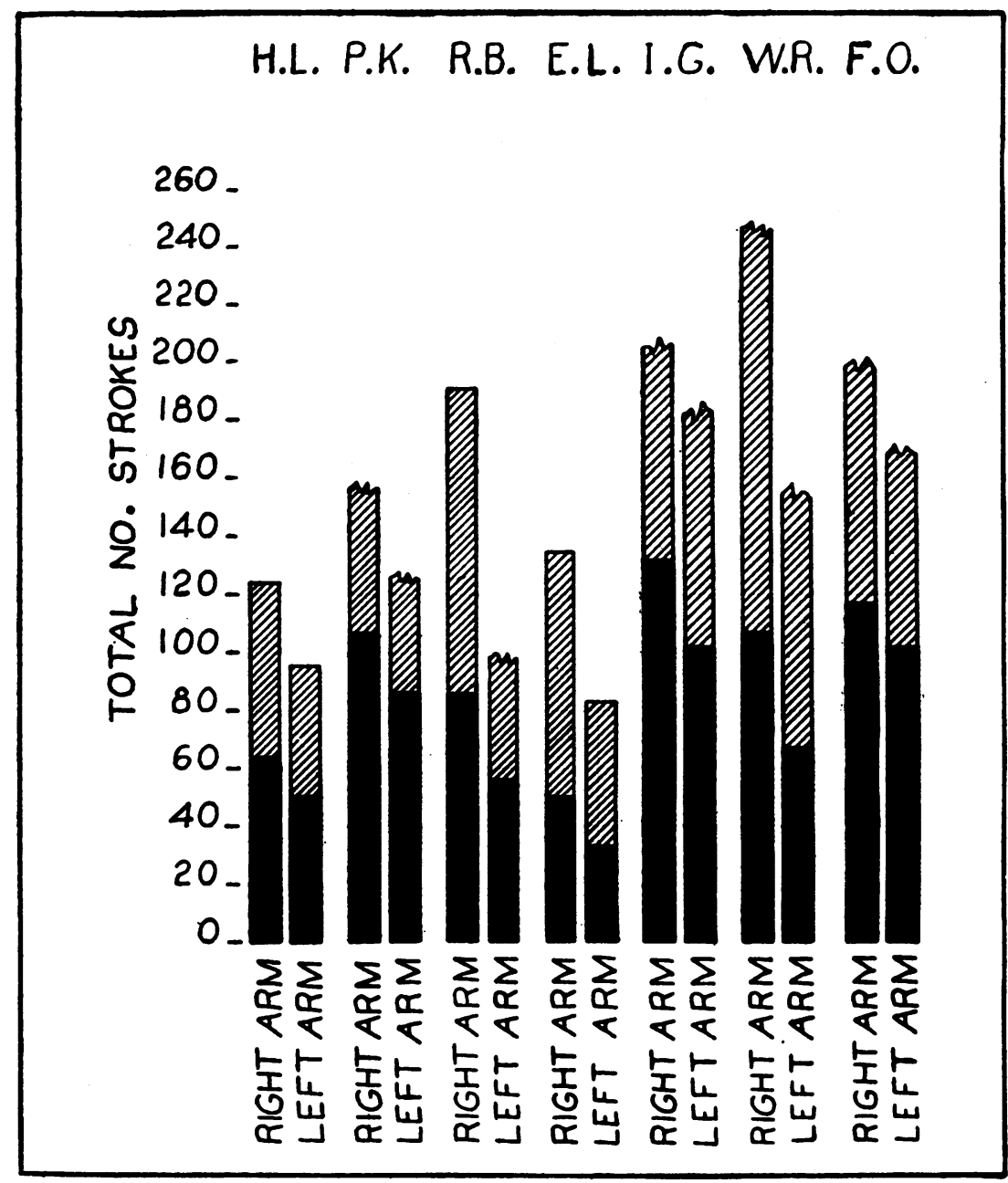

Fig. 8. Graphic Summary of Results in Seven Normal Subjects Showing the Difference in the Amount of Exercise Reguired to Cause Pain in The Right AND LEFT ARMS.

Exercise was carried out as in experiments shown in Figure 1; exercise was at a rate of 60 contractions per minute and the cuff pressure was at $160 \mathrm{~mm}$. $\mathrm{Hg}$. The exercises of the two arms were carried out within 30 to 45 minutes of each other. The significance of the blocks etc. is as in Figure 1. Discussed in text.

more frequently with the right hand than with the left in all of these subjects.

The manner in which this difference in onset of pain and fatigue is brought about is not entirely clear. Several possibilities exist. The difference may be due to a difference in the threshold of the end-organs for pain and of the myoneural junctions and effector nerve endings, respectively, or to the difference in mechanical efficiency with which the trained right arm and untrained left do their exercises. Another possibility suggested by our results with hypercapnia and ingestion of $\mathrm{NaHCO}_{3}$ is that the difference lies in the buffering capacity of the two muscles. There is evidence to suggest that the buffering power of muscles is increased with training (11).

The importance of avoiding unnecessary rest in patients subject to intermittent claudication or to anginal pain is evident from these results, and the importance of training in such patients is obvious. There is no doubt that if training is cautiously carried out the patient will be better able 
to meet some of the demands which arise unavoidably in emergencies even when activity is limited.

\section{SUM MARY}

1. The main findings in this study with regard to the amount of exercise required to cause pain and fatigue in the muscles of the arm of normal subjects are:

(a) Circulatory slowing caused a decrease in the amount of exercise required to cause pain, the effect being disproportionately greater at high degrees of circulatory slowing. The amount of blood trapped in the arm played an insignificant rôle. Circulatory slowing had its greatest effect at the fastest rates of exercise.

(b) Increasing the rate of exercise led to a decrease in the amount of exercise required to cause pain when the circulation to the limb was unobstructed. Slowing the circulation led to a diminution of this effect of rate of exercise. This effect of rate of exercise disappeared when the circulation in the limb was stopped.

(c) Exercise of a large group of muscles of the leg to the point of pain had a two-fold action on the amount of exercise required to cause pain in a subsequent arm-exercise; viz., (1) by an action on the central nervous system it augmented the amount of exercise required to cause pain and (2) by an action through transport of blood from the exercised legs to the muscles of the arm it decreased the amount of exercise required to cause pain. Special procedures were required to separate these two effects.

(d) Increasing the $\mathrm{CO}_{2}$ content of the blood in the arm decreased the amount of exercise required to cause pain. Ingestion of large amounts of sodium bicarbonate increased the amount of exercise required to cause pain. Ingestion of sodium bicarbonate also tended to alleviate the pain of patients with intermittent claudication and of those with angina pectoris.

(e) In right handed subjects less exercise was required to cause pain in the left than in the right arm.

(f) The effects of these procedures on neuromuscular fatigue were not related quantitatively to their effects on pain. With certain procedures, such as the ingestion of sodium bicarbonate, the effects were opposite.
2. The significance of these results on pain and fatigue in muscle is discussed in the body of the paper. The salient conclusions from these experiments are:

(a) The time allowed for recovery between contractions in a rhythmically contracting muscle alters the rate of accumulation of the substance(s) leading to pain, implying that the pain-producing substance(s) is a product of metabolic muscular activity.

(b) The substance(s) causing pain diffuses into and out of the blood stream. It is nonvolatile since it operates even after passing through the lungs.

(c) The appearance of pain in muscle is dependent not only upon the local production of pain-producing substance(s) but to a certain extent upon the transport of such a substance(s) from other regions.

(d) This non-volatile pain-producing substance(s) appears to be acid in character; at least its action is facilitated by acid and retarded by alkaline substances.

(e) Acids and bases exhibit summation of effect with the pain-producing substance(s) by changing the $\mathrm{pH}$ of the end organs, by altering their buffering capacity, or in both ways.

( $f$ ) Training tends to lessen the action of the pain-producing substance(s), probably by altering the buffering capacity of the muscle concerned.

(g) The variability in the appearance of fatigue, which is independent of pain, plays an important rôle in forestalling the appearance of pain under certain circumstances.

3. The bearing of these findings on the appearance of pain in angina pectoris and in intermittent claudication is discussed at the end of each section.

We are grateful to the volunteers, members of the Department, who undertook these exercises for us.

\section{BIBLIOGRAPHY}

1. Lewis, T., Pickering, G. W., and Rothschild, P., Observations upon muscular pain in intermittent claudication. Heart, 1931, 15, 359.

2. Lewis, $T$., Pain in muscular ischemia; its relation to anginal pain. Arch. Int. Med., 1932, 49, 713. 
3. Kissin, M., The production of pain in exercising skeletal muscle during induced anoxemia. J. Clin. Invest., 1934, 13, 37.

4. Perlow, S., Markle, P., and Katz, L. N., Factors involved in the production of skeletal muscle pain. Arch. Int. Med., 1934, 53, 814.

5. Katz, L. N., Mechanism of pain production in angina pectoris. Am. Heart J., 1935, 10, 322.

6. Hill, A. V., Muscular movement in man. McGrawHill Book Co., Inc., New York, 1927.

7. MacWilliam, J. A., and Webster, W. J., Some applications of physiology to medicine. I. Sensory phenomena associated with defective blood supply to working muscles. Brit. M. J., 1923, 1, 51.
8. Reid, C., Experimental ischemia : sensory phenomena, fibrillary twitchings and effects on pulse, respiration, and blood pressure. Quart. J. Exper. Physiol., 1931, 21, 243.

9. Laplace, L. B., and Crane, M. P., Observations on the production of pain and fatigue in muscular ischemia and their relationship in angina pectoris. Am. J. M. Sc., 1934, 187, 264.

10. Moore, R. M., Moore, R. E., and Singleton, A., Jr., Experiments on the chemical stimulation of painendings associated with small blood-vessels. Am. J. Physiol., 1934, 107, 594.

11. Ferrari, R., Zur Frage des Muskeltrainings. Arch. f. d. ges. Physiol., 1932, 230, 639. 